\title{
Aspectos reprodutivos do caranguejo Aratus pisonii (H. Milne Edwards) (Crustacea, Decapoda, Grapsidae) do manguezal de Itacuruçá, Rio de Janeiro, Brasil
}

\author{
Cristiane Ferreira Nicolau ${ }^{1,2}$ \\ Lídia Miyako Yoshii Oshiro ${ }^{2}$
}

\begin{abstract}
Reproductive aspects of crab Aratus pisonii (H. Milne Edwards) (Crustacea, Decapoda, Grapsidae) in Itacuruçá's mangrove. The objective of this work has to get knowledge about the reproductive aspect of the tree crab Aratus pisonii (H. Milne Edwards, 1837). The sampling were made monthly from April, 2000 to April, 2001, in the Itacuruçá's mangrove $\left(22^{\circ} 54^{\prime} \mathrm{S}\right.$ and $\left.43^{\circ} 33^{\prime} \mathrm{W}\right)$, Rio de Janeiro. The crabs were collected handly, using an effort of 50 minutes with three collectors. In laboratory, the specimens were separated by sex and had measured the carapace width and lenght. The ovigerous females were separated and the mass of eggs was removed for a mensurament of size and count of eggs. Afterwards, the animals were conservad in $70 \%$ alcohol. A total of 783 individuals were caught among $59 \%$ males and $41 \%$ females among these $8 \%$ were ovigerous. The ovigerous females were found almost during the whole year, except in the winter showing major plenty on February and March, demonstrating the reproductive period. The ovigerous females presented size between 14.0 and $23.6 \mathrm{~mm}$ of carapace width and the average fecundity estimated to the specie was $9081 \pm 3684$ eggs. The mean size of the eggs was $0.27 \mathrm{~mm}$ varied from 0.24 to $0.29 \mathrm{~mm}$ and the size at onset of egg production was verified from 13.1 to 15.0 $\mathrm{mm}$ of the carapace width.
\end{abstract}

KEY WORDS. Aratus pisonii, reproduction, tree crab, Itacuruçá's mangrove, Sepetiba Bay

O litoral do estado do Rio de Janeiro é bastante recortado e composto por várias baías e enseadas, destacando-se entre elas a Baía de Sepetiba. A Baía de Sepetiba é importante por suas atividades de pesca e de turismo, comportando um importante manguezal da cidade de Itacuruçá município de Mangaratiba.

$\mathrm{Na}$ Baía de Sepetiba, encontram-se os manguezais que constituem ecossistemas de transição, típicos de regiões tropicais e subtropicais, ocorrendo em zonas costeiras. É um ecossistema propício para reproduçãoe alimentação de muitas espécies animais, sendo considerado transformadores de nutrientes em matéria orgânica (SCHAEFFER-NOVELLI 1995). O manguezal da Baía de Sepetiba, abriga diversas espécies de crustáceos, principalmente os caranguejos da família Grapsidae, representada por cinco espécies: Goniopsis cruentata (Latreille,1803); Aratus pisonii (H. Milne Edwards, 1837); Sesarma rectum Randall, 1840; Chasmagnatus granulata Dana, 1851; Metasesarma rubripes (Rathbun, 1897) (OSHIRO et al. 1998).

1) Curso de Pós-Graduação em Biologia Animal, Universidade Federal Rural do Rio de Janeiro.

2) Estação de Biologia Marinha, Universidade Federal Rural do Rio de Janeiro. Rua Sereder, Itacuruçá, 23880-000 Rio de Janeiro, Rio de Janeiro, Brasil. 
O caranguejo A. psonii é muito abundante e habita principalmente a borda e o meio do manguezal (OSHIRO et al. 1998). É um animal pequeno, possui hábito arborícola, vivendo em ramos e troncos do mangue (WARNER 1967). Segundo BEEVER et al. (1979), essa espécie possui papel importante na cadeia alimentar, sendo considerado herbívoro primário e predadores de pequenos artrópodes.

Aratus pisonii é popularmente conhecido como marinheiro e apresenta uma ampla distribuição geográfica, ocorrendo no Atlântico Ocidental da Flórida, Golfo do México, Antilhas, Norte da América do Sul, Guianas e Brasil (do Piauí até o estado do Paraná), Pacífico Oriental - da Nicarágua até o Peru (COELHO \& RAMOS 1972; MELO 1996).

CoELHO (1966), verificou que além de subir em ramos e troncos do mangue, esses caranguejos podem ser encontrados em rios e todos os regimes.

No Brasil, informações acerca da biologia reprodutiva dessa espécie tem sido obtida no estado de São Paulo em relação à fecundidade e os modos de reprodução (LEME \& Negreiro-Fransozo 1998a, b), mas no estado do Rio de Janeiro, há poucas informações sobre essa espécie, somente alguns trabalhos realizados por LACERDA $(1981,1991)$, relacionados aos hábitos alimentares. Portanto, este trabalho teve como objetivo contribuir para o conhecimento sobre o período reprodutivo, a fecundidade, o tamanho dos ovos e a maturidade sexual fisiológica de fêmeas $A$. pisonii no manguezal de Itacuruçá, Rio de Janeiro.

\section{MATERIAL E MÉTODOS}

As coletas foram realizadas mensalmente de abril de 2000 a abril de 2001, no manguezal de Itacuruçá, localizado no município de Mangaratiba, entre as coordenadas $22^{\circ} 54^{\prime}$ S e $43^{\circ} 33^{\prime} \mathrm{W}$ na Baía de Sepetiba, Rio de Janeiro.

Os caranguejos foram capturados manualmente, durante períodos de marés baixas de acordo com a tabela de marés do Porto de Sepetiba (DHN 2000 2001), utilizando esforço de captura de 50 minutos com auxílio de três coletores.

$\mathrm{O}$ material coletado foi devidamente etiquetado e mantido em freezer até o processamento das análises. Após o descongelamento, o processamento consistiu na determinação do sexo e mensuração das seguintes partes dos animais: comprimento da carapaça (CC), largura da carapaça (LC) e largura do abdome (LA). Essas medidas foram tomadas com auxílio de um paquímetro com precisão de $0,1 \mathrm{~mm}$.

Os animais foram distribuídos em 10 classes de tamanhos com intervalos de $2 \mathrm{~mm}$ de acordo com a largura da carapaça (LC), variando de 5,1 a $25,0 \mathrm{~mm}$.

$\mathrm{O}$ período reprodutivo foi determinado através da frequiência mensal de fêmeas ovígeras durante todo o período amostrado.

Para estimar a fecundidade das fêmeas ovígeras, os pleópodos com a massa de ovos em todos os estágios de desenvolvimento, foram retirados e os ovos foram dissociados, utilizando-se a solução de hipoclorito de sódio (água sanitária comercial), durante alguns segundos tomando-se o cuidado para que os ovos não sofressem ruptura. A seguir foram lavados em água corrente, sendo colocados em um béquer, completando o volume com água a $80 \mathrm{ml}$. A amostra foi homogeneizada e foram tomadas três sub-amostras de $1 \mathrm{ml}$, onde foram contados o número de ovos, utilizando-se um contador manual sob um microscópio estereoscópico. 
O tamanho dos ovos foi obtido através de uma ocular de medição do microscópio estereoscópico, com aumento de 25 vezes, fazendo-se a medição aleatória de 20 ovos de cada fêmea ovígera.

As fêmeas foram dissecadas e as gônadas observadas macroscopicamente e, de acordo com a coloração e espessura, foram identificados os estádios gonadais. Posteriormente, os animais foram conservados em álcool a 70\%.

O tamanho mínimo da primeira maturação sexual fisiológica foi estimado segundo VAZZOLER (1981), considerando fêmeas adultas ou jovens de acordo com o estádio gonadal apresentado. Foram consideradas fêmeas imaturas aquelas que apresentaram gônadas finas e transparentes e fêmeas maturas aquelas apresentando gônadas visíveis variando da coloração amarelo ao laranja vivo, ou ovários flácidos e transparentes após a desova.

Os procedimentos laboratoriais foram realizados na Estação de Biologia Marinha (EBM) da Universidade Federal Rural do Rio de Janeiro (UFRRJ).

\section{RESULTADOS E DISCUSSÃO}

Foram coletados um total de 783 indivíduos, sendo $59 \%(\mathrm{n}=462)$ machos e $41 \%(n=321)$ fêmeas, das quais $8 \%(n=62)$ fêmeas do total eram ovígeras.

A largura do carapaça das fêmeas não ovígeras variou de 5,1 a $25,0 \mathrm{~mm}$, enquanto nos machos variou de 7,1 a $25,0 \mathrm{~mm}$. As fêmeas foram mais frequentes entre as classes de tamanho de 17,1 a 19,0 mm e os machos entre as classes de 19,1 a 21,0 mm (Fig. 1).

No presente estudo largura do carapaça das fêmeas ovígeras variou de 14 a 23,6 mm (Fig. 1), entretanto DiAZ \& CONDE (1989) encontraram na Venezuela, fêmeas ovígeras variando de 11 a $24 \mathrm{~mm}$. Segundo estes autores, $A$. pisonii alcança diferentes tamanhos de acordo com as condições estruturais do manguezal, pois é um ecossistema de grande produtividade primária (CONDE \& DIAZ 1989).

A maior freqüência de fêmeas ovígeras ocorreu durante os meses de fevereiro e março. Nos meses de maio a julho não foram capturadas fêmeas ovígeras (Fig. 2). DiAZ \& CONDE (1989) na Venezuela e WARNER (1967) na Jamaica, encontraram reprodução contínua em A. pisonii, durante todo o ano. A duração do período reprodutivo pode ser determinada pelas condições ambientais favoráveis de forma que as variações intraespecíficas relacionadas com a periocidade reprodutiva podem ocorrer como respostas adaptativas para que o sucesso reprodutivo seja alcançado nos diferentes ambientes (SASTRY 1983).

A fecundidade média estimada no presente estudo foi de $9081 \pm 3684$ ovos, variando de 2320 a 20720 ovos (Fig. 3). A equação que descreve a relação entre o número de ovos carregados pelas fêmeas ovígeras e a largura da carapaça é $\mathrm{F}=$ 990,39 LC - $10682(\mathrm{R}=0,61)$. Verifica-se que o coeficiente de correlação é baixo, mas o número de ovos aumenta em função da largura da carapaça (Tab. I), como ocorre em outros braquiúros. A grande variação na fecundidade observada neste trabalho, pode estar relacionado à perda dos ovos durante a incubação, devido às atividades mecânicas dos caranguejos (HINES 1982; WOLCOTT \& WOLCOTT 1982), ou ainda à ocorrência de desovas múltiplas (WEAR 1974). 

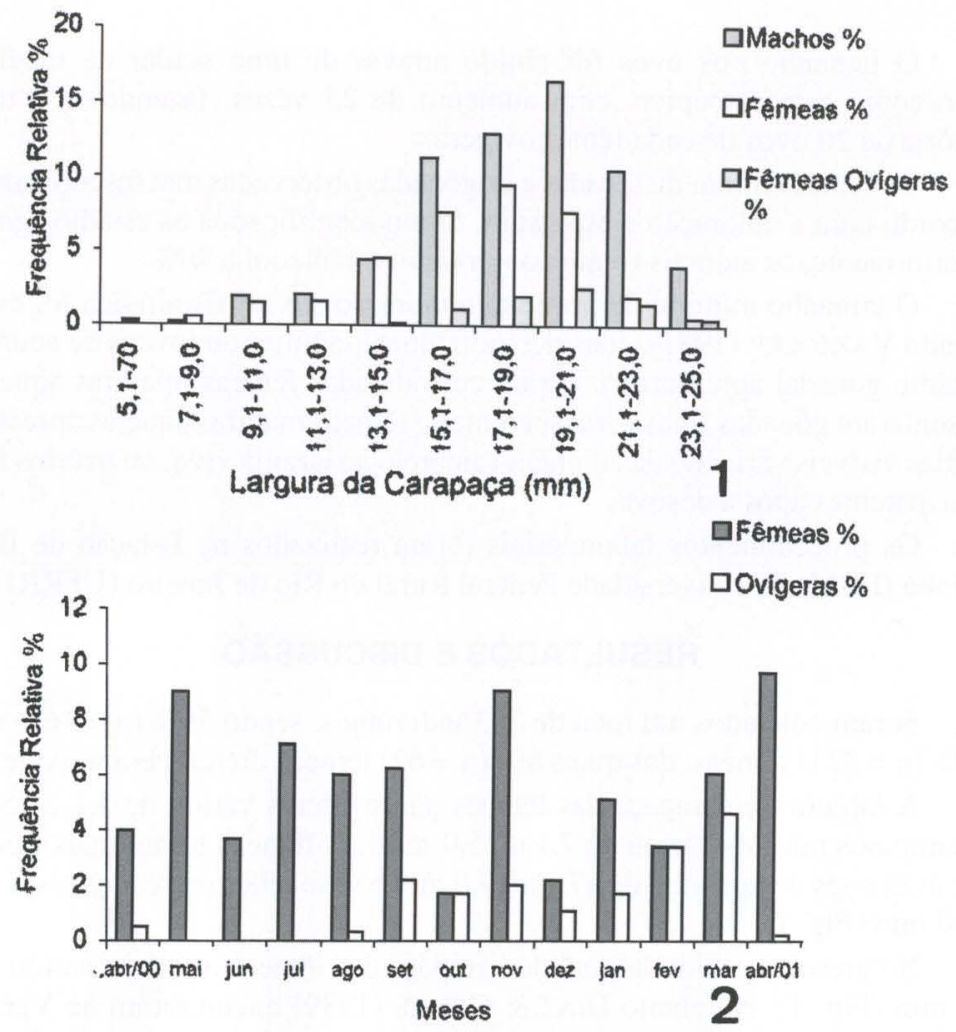

Figs 1-2. Aratus pisonii, distribuição da freqüência relativa de: (1) machos, fêmeas não ovígeras e fêmeas ovígeras de acordo com a largura da carapaça, (2) fêmeas não ovígeras e ovígeras, durante o período de abril de 2000 a abril de 2001, coletados no manguezal de Itacuruçá, Rio de Janeiro.

Tabela I. Aratus pisonii. Fecundidade média relacionada com a largura da carapaça, de fêmeas ovígeras coletadas no período de abril/2000 a abril/2001, no manguezal de Itacuruçá, Rio de Janeiro.

\begin{tabular}{cccc}
\hline Largura da Carapaça $(\mathrm{mm})$ & Variação do número de ovos & Fecundidade média & Desvio Padrão \\
\hline $13,1-15,0$ & 7280 & 7280 & - \\
$15,1-17,0$ & $2426-10533$ & 5359 & 2975,2 \\
$17,1-19,0$ & $2320-16853$ & 6506 & 2754,4 \\
$19,1-21,0$ & $3040-16373$ & 9488 & 3341,9 \\
$21,1-23,0$ & $3840-20720$ & 11262 & 2036,9 \\
$23,1-25,0$ & $12080-17093$ & 13902 & 2772,8 \\
\hline
\end{tabular}

De acordo com a tabela II, verifica-se que a fecundidade média obtida no presente trabalho foi superior ao encontrado por WARNER (1967) e inferior aquelas obtidos por, DiaZ \& CONDE (1989), CONDE \& DiAZ (1989) e LEME \& NEGREIROFRANSOZO (1998a). Segundo esses autores, a quantidade de ovos varia segundo o peso e a largura da carapaça. 

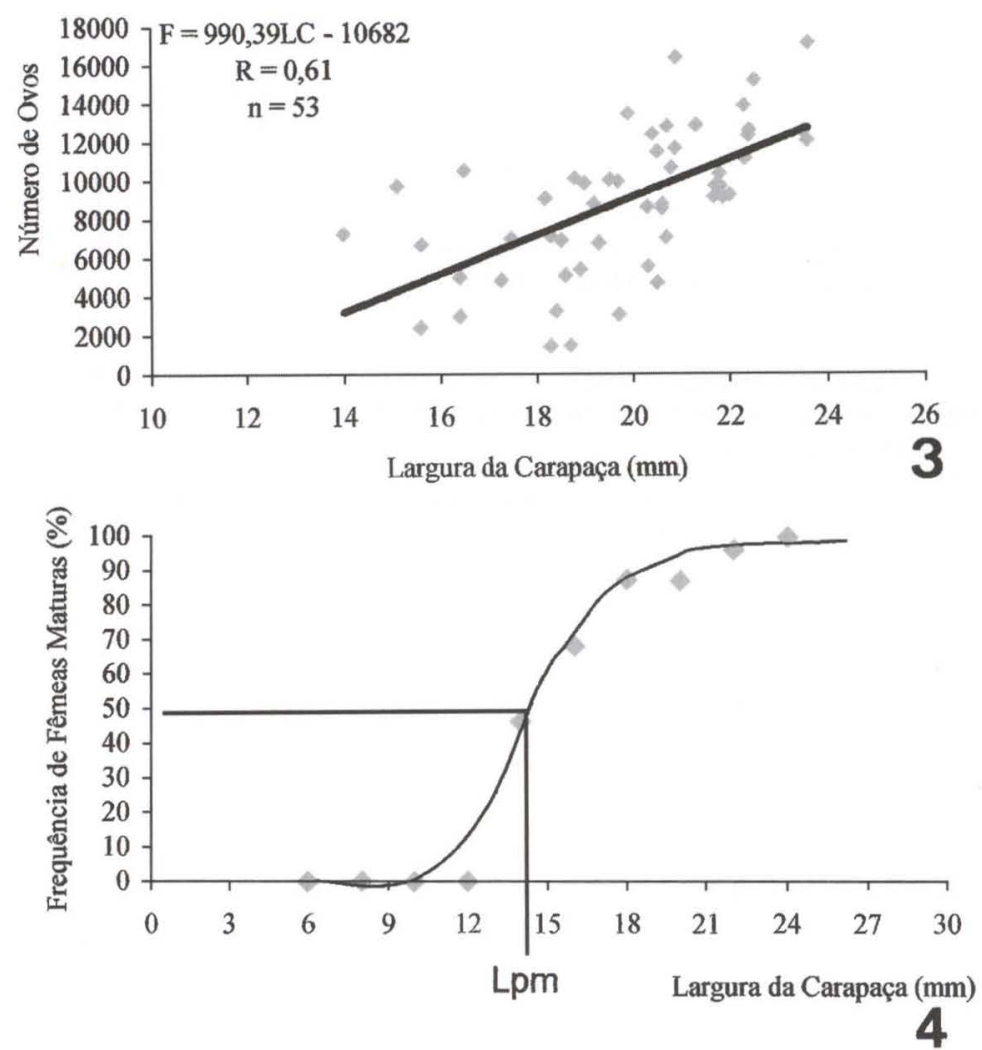

Figs 3-4. Aratus pisonii:: (3) distribuição do número de ovos das fêmeas ovigeras e (4), distribuição de freqüência relativa de fêmeas maduras, de acordo com a largura da carapaça, coletados no período de abril de 2000 a abril de 2001, no manguezal de Itacuruçá, Rio de Janeiro.

O diâmetro dos ovos em todos os estágios de desenvolvimento, neste estudo variou de 0,24 a $0,29 \mathrm{~mm}$ apresentando um diâmetro médio de $0,27 \mathrm{~mm} \pm 0,01$. De acordo com a tabela II, este resultado apresentou-se inferior aos obtidos por WARNER (1967). Essa diferença no tamanho dos ovos não é muito freqüente, mas diferenças intraespecíficas podem ocorrer devido as diferenças na latitude (EFFORD 1969; JONES \& SiMONS 1983), as diferentes estações do ano (DiAZ 1980) e também pode estar relacionada com a temperatura, salinidade e com a disponibilidade de alimento para as fêmeas adultas, ou uma combinação de todos esses fatores (RABALIS \& GORE 1985).

O tamanho médio da maturação sexual fisiológica das fêmeas foi estimado para a classe de tamanho de 13,1 a 15,0 mm de largura da carapaça (Fig, 4). HARTNOLL (1965), obteve tanto a maturidade fisiológica como a morfológica para o tamanho de 15 a 17 mm. Já WARNER (1967), encontrou fêmeas de A. pisonii incubando ovos com $12 \mathrm{~mm}$ de largura da carapaça, enquanto DIAZ \& CONDE 
(1989), encontraram a menor ovígeras com $11,3 \mathrm{~mm}$ de largura da carapaça. As variações de latitude e os fatores ambientais, tais como a temperatura e a interação entre temperatura -fotoperíodo, influenciam o tamanho da maturidade sexual (GIESE 1959; JONES \& SiMONS 1983). Tais fatores, podem interferir no metabolismo e no crescimento em populações de diferentes regiões, originando intervalos de tamanhos diferentes para o início da maturação em uma mesma espécie como observado para Pachygrapsus crassipes (HINES 1989).

Tabela II. Largura da Carapaça, variação no tamanho dos ovos, diâmetro médio dos ovos e fecundidade média de Aratus pisonii de acordo com diferentes autores, ocorrente em manguezais de diferentes locais.

\begin{tabular}{|c|c|c|c|c|c|}
\hline Autor & Local & $\begin{array}{l}\text { Largura da carapaça } \\
\qquad(\mathrm{mm})^{*}\end{array}$ & $\begin{array}{l}\text { Variação no tamanho } \\
\text { dos ovos }(\mathrm{mm})\end{array}$ & $\begin{array}{c}\text { Tamanho } \\
\text { médio dos ovos }\end{array}$ & $\begin{array}{l}\text { Fecundidade } \\
\text { média }\end{array}$ \\
\hline WARNER (1967) & Jamaica & 23,0 & $0,24-0,35$ & - & 4900 \\
\hline DIAZ \& CONDE (1989) & Venezuela & 23,1 & - & - & 11577 \\
\hline CONDE \& DIAZ (1989) & Venezuela & 26,3 & - & - & 16379 \\
\hline $\begin{array}{l}\text { LEME \& NEGREIRO- } \\
\text { FRANSOZO (1998 a) }\end{array}$ & Brasil & 24,3 & - & - & 15197 \\
\hline Presente estudo & Brasil & 23,6 & $0,24-0,29$ & 0,27 & 9081 \\
\hline
\end{tabular}

* Tamanho máximo de largura da carapaça.

AGRADECIMENTOS. Agradecemos a colaboração do Sr. Casemiro Antonio Alves da Estação de Biologia Marinha da UFRRJ, no auxílio às coletas mensais e das Ms. Zilanda de Souza Silva e Giovana Valverde Lima nas coletas, utilização dos programas para análise e apresentação dos dados.

\section{REFERÊNCIAS BIBLIOGRÁFICAS}

Beever III, J.W.; D. Simberloff \& L.L. King. 1979. Herbivory and predation by the Mangrove tree crab Aratus pisonii. Oecologia 43: 317-328.

CoELho, P. A. 1965. Os crustáceos decápodas de alguns manguezais pernambucanos. Trab. Oceanogr. Univ. Fed. Pernambuco, Recife, 7/8: 71-90.

Coelho, P.A. \& M.A. Ramos. 1972. A constituição e a distribuição da fauna de decápodos do litoral leste da América do Sul, entre as latitudes $5^{\circ} \mathrm{Ne} 39^{\circ} \mathrm{S}$. Trab. Oceanogr. Univ. Fed. Pernambuco, Recife, 13: 133-236.

CondE, J.E. \& H. DíAZ. 1989. The mangrove tree crab Aratus pisonii in a tropical estuarine coastal lagoon. Estuar. Cost. Shelf. Sci., London. 28: 639-650.

Dhn. 2000. Tábua de marés para o ano 2000, costa do Brasil e Portos Estrangeiros. Rio de Janeiro, Diretoria de Hidrografia e Navegação da Marinha do Brasil, p. 117-119.

— . 2001. Tábua de marés para o ano 2001, costa do Brasil e Portos Estrangeiros. Rio de Janeiro, Diretoria de Hidrografia e Navegação da Marinha do Brasil, p. 120-122.

DíAz, H. 1980. The mole crab Emerita talpoida (Say); a case of changing life history pattern Ecol. Monogr. 50 (4): 437-456.

DíAZ, H. \& J.E. CONDE. 1989. Populations dynamics and life history of the mangrove crab Aratus pisonii (Brachyura: Grapsidae) in a marine environment. Bull. Mar. Sci., Miame, 45 (1): 148-163.

EFFORD, I.E. 1969. Egg size in the sand crab, Emerita analoga (Decapoda- Hippidae). Crustaceana, Netherlands, 16: 293-308

GIESE, A.C. 1959. Comparative physiology: annual reproductive cycles of marine Invertebrates. Ann. Rev. Physiol. 21: 547-576. 
Hartnoll, R.G. 1965. Notes on the marine grapsid crabs of Jamaica. Proc. Linn.. Soc. Lond. 176 (2): 113-147.

HINES, A.H. 1982. Allometric constraints and variables of reproductive effort in brachyuran Crabs. Mar. Biol., Heidelberg, 69: 309-320.

- 1989. Geographic variation in size at maturity in brachyuran crabs. Bull. Mar. Sci., Miami, 42 (2): 356-368.

JONES, M.B. \& M.J. SimONS. 1983. Latitudinal variation in reproductive characteristics of a mud crab Helice grassa (Grapsidae). Bull. Mar. Sci., Miami, 33 (3): 656-670.

LACERDA, L.D. 1981. Mangrove wood pulp, an alternative food source for the tree-crab Aratus pisonii. Biotropica, Washinghton, DC, 13 (4): 137.

LaCerda, L.D.; C.A.R. Silva; C.E. RezendE \& L.A. MarTinelli. 1991. Food sources for the mangrove tree crab Aratus pisonii: a carbon isotopic study. Rev. Bras. Biol., Rio de Janeiro, 51 (3): 685-687

Leme, M.H. De A. \& M.L. Negreiro-Fransozo. 1998a. Fecundity of Aratus pisonii (Decapoda: Grapsidae) in the Ubatuba region, State of São Paulo, Brazil. Iheringia. Sér. Zoo., Porto Alegre, (84): 73-77.

-1998b. Reproductive patterns of Aratus pisonii (Decapoda: Grapsidae) from estuarine area of São Paulo Northern Coast, Brazil. Rev. Biol. Trop., San José, 46 (3): 673-678.

MeLo, G.A.S. 1996. Manual de Identificação dos Brachyura (Caranguejos e Siris) do Litoral Brasileiro. São Paulo, Ed. Plêiade, FAPESP, Museu de Zoologia, 603p.

Oshiro, L.M.Y.; R. Silva \& Z.S. Silva. 1998. Composição da fauna de braquiúros (Crustacea: Decapoda) da Baía de Sepetiba - RJ. Náuplius, Rio Grande, 6: 31-40.

RaBALAIS, N.N. \& R.H. GORE. 1985. Abbreviated development in decapods, p. 67-126. In: A.M. WERNER (Ed.). Larval growth. Boston, A.A. Balkema, 236p.

SASTRY, A.N. 1983. Ecological aspects of reproduction, p. 179-269. In: F.J. VernenberG \& W.B. Vernenberg (Eds). The Biology of Crustacea. Environmental adaptations. New York, Academic Press, vol. 8,383p.

SCHAEFfer-Novelli, Y. 1995. Manguezal Ecossistema entre a terra e o mar. São Paulo, EDUSP, 64p.

VAzzoler, A.E.M. 1981. Manual de metódos para estudos biológicos de populações de peixes, reprodução e crescimento de peixes. Brasília, CNPq, Programa Nacional de Zoologia, 108p.

WARNER, G.F. 1967. The life history of the mangrove tree crab Aratus pisoni. Jour. Zool. London 153: 321-335.

WEAR, R.G. 1974. Incubation in British decapod Crustacea, and the effects of the temperature on the rate and sucess of embryonic development. Jour. Mar. Biol. Ass. U.K. 54: 745-762.

WolcotT, T.G. \& D.L. WolcotT. 1982. Larval loss and spawning behavior in the land crab Gecarcinus lateralis (Fréminville). Jour. Crustacean Biol. 2 (4): 477-485.

Recebido em 08.VIII.2002; aceito em 27.XI.2002. 